low-density lipoprotein cholesterol (LDL-C) levels. 22,1\% had arterial hypertension $(\mathrm{AH}), 29,5 \%$ were current smokers, $27,4 \%$ had excess body weight, $3,1 \%$ had family history of cardiovascular diseases (CVD). Traditional risk factors for atherosclerosis were found in $80 \%$ out of 40 gender and age matching subjects from the control group, i.e., showing practically the same prevalence as in BD pts.

Table 1. The incidence-rate of traditional risk factors in BD patients and controls

\begin{tabular}{lccc}
\hline Variables & BD pts $(\mathrm{n}=95)$ & Controls $(\mathrm{n}=40)$ & $\mathrm{p}$ \\
\hline Age, years & $29,0[23,0 ; 35,0]$ & $29,0[25,0 ; 32,0]$ & $\mathrm{NS}$ \\
$\mathrm{BMI}, \mathrm{kg} / \mathrm{m}^{2}$ & $23,1[21,1 ; 25,5]$ & $23,8[22,0 ; 26,0]$ & $\mathrm{NS}$ \\
$\mathrm{BMI} \geq 25 \mathrm{~kg} / \mathrm{m}^{2}, \mathrm{n}(\%)$ & $26(27,4)$ & $15(37,5)$ & $\mathrm{NS}$ \\
Arterial hypertension, $\mathrm{n}(\%)$ & $21(22,1)$ & $4(10)$ & $\mathrm{NS}$ \\
Family history of CVD & $3(3,1)$ & $5(12,5)$ & 0,05 \\
Cigarette smoking, $\mathrm{n}(\%)$ & $28(29,5)$ & $12(30,0)$ & $\mathrm{NS}$ \\
Dyslipidemia, $\mathrm{n}(\%)$ & $73(76,8)$ & $27(67,5)$ & $\mathrm{NS}$ \\
Total cholesterol, mml/l & $5,0[4,3 ; 6,1]$ & $5,1[4,5 ; 5,7]$ & $\mathrm{NS}$ \\
Cholesterol $>5,0 \mathrm{mml} / \mathrm{l}, \mathrm{n}(\%)$ & $47(49,5)$ & $25(62,5)$ & $\mathrm{NS}$ \\
TGs, mml/l & $0,92[0,7 ; 1,3]$ & $0,89[0,60 ; 1,08]$ & $\mathrm{NS}$ \\
TGs $>1,7 \mathrm{mml} / \mathrm{l}, \mathrm{n}(\%)$ & $8(8,4)$ & $3(7,5)$ & $\mathrm{NS}$ \\
LDL, mml/l & $3,36(2,8 ; 4,0)$ & $3,3[2,7 ; 3,7]$ & $\mathrm{NS}$ \\
LDL $>3 \mathrm{mml} / \mathrm{l}, \mathrm{n}(\%)$ & $63(66,3)$ & $25(62,5)$ & $\mathrm{NS}$ \\
$\mathrm{HDL}, \mathrm{mml} / \mathrm{l}$ & $1,19[1,0 ; 1,5]$ & $1,35[1,2 ; 1,6]$ & 0,06 \\
$\mathrm{HDL} \leq 1 \mathrm{mml} / \mathrm{l}(\mathrm{male}), \leq 1,2 \mathrm{mml} / \mathrm{l}$ (female) $\mathrm{n}(\%)$ & $30(31,6)$ & $5(12,5)$ & 0,03 \\
\hline
\end{tabular}

Reduced HDL levels were more common in BD pts vs the controls - $30(31,6 \%)$ vs $5(12,5), p=0,03$ as shown in table 1 . Other traditional risk factors were similarly present in both groups with no significant difference between BD pts and the controls.

Analysis showed similar incidence of CV events (nonfatal myocardial infarction, angina pectoris and stroke) in both groups. There were no differences between BD pts and healthy people in terms of lifetime risk for CVD. High lifetime risk $\geq$ $20 \%$ was found in 4 BD pts vs 0 in the control group; moderate lifetime risk $>10 \%$ $<20 \%$ - in $6,3 \%$ of BD pts vs $2,5 \%$ in the control group, and low lifetime risk < $10 \%$-was found in $89,5 \%$ of BD pts vs $97,5 \%$ of the controls.

Average IMT values were significantly higher in BD pts $-0.68[0.60 ; 0.73]$ vs 0.63 [0.56;0.65], $p=0.008$, but at the same time, IMT thinning (up to $<0.5 \mathrm{~mm}$ ) was significantly more common in BD pts $-32.6 \%$ vs $12.5 \%$ in the control group $(p=0.01)$. HsCRP serum levels were significantly higher in BD pts $(2.42[0.5 ; 8.8]$ $\mathrm{mg} / \mathrm{I})$ than in the control group $(0.37[0.15 ; 0.75]), p=0.001$. There was a correlation between hs-CRP and BMI in BD pts $(r=0.2, p<0.05)$, but no correlation was found between hsCRP and BD activity/BD clinical manifestations.

Conclusion: Decreased HDL cholesterol levels were more common in BD patients than in the control group, just as thinning of IMT, most likely because of vasculitis.

Disclosure of Interests: None declared

DOI: 10.1136/annrheumdis-2021-eular.3431

\section{POS0116 HEART RATE VARIABILITY IN PATIENTS WITH BEHCETS DISEASE}

R. Goloeva ${ }^{1}$, Z. Alekberova ${ }^{1}$, T. Popkova ${ }^{1}$, S. Glukhova ${ }^{1}$, D. Novikova ${ }^{1}$. ${ }^{1} V$ A Nasonova Research Institute of Rheumatology, Systemic Rheumatic Diseases, Moscow, Russian Federation

Background: Behcet's disease (BD) is a systemic vasculitis affecting all types and sizes of blood vessels. Heart rate variability (HRV) reflects sympathetic -parasympathetic imbalance in the autonomic NS regulation. Low HRV values are known as independent risk factor of death and non-fatal cardiovascular events in both - survivors of a myocardial infarction and in asymptomatic population. Objectives: The aim of this study is to evaluate HRV in BD pts vs healthy controls.

Methods: The study group included $74 \mathrm{BD}$ pts (53males/21females) with disease duration of $9,0(5,0 ; 15,0) / 9,0(7 ; 20)$ years, and the control group - 32/15 age-matched healthy $\mathrm{m} / \mathrm{f}$. The following HRV parameters from $24 \mathrm{~h}$ ECG ambulatory recording were assessed: MeanNN and time-domain variables, adjusted by MeanNN (SDNNn\%, SDNNin\%, RMSSDn\%). Additionally, all traditional cardiovascular risk factors such as systolic blood pressure (SPB), smoking status, BMI values, dyslipidemia profile, ultrasonographic values of carotid intima-media thickness (IMT), and levels high sensitive CRP (hsCRP) as a marker of inflammation were evaluated.

Results: In BD patients HRV values (RMSSDn\%) were significantly lower compared to healthy controls (table 1 ).
Table 1. HRV parameters in BD patients and control group

\begin{tabular}{lcccc}
\hline Parameters & \multicolumn{2}{c}{ Males } & \multicolumn{2}{c}{ Females } \\
\hline & BD $(n=53)$ & Control $(n=32)$ & BD $(n=21)$ & Control $(n=15)$ \\
\hline Age, years & $30(24 ; 36)$ & $30(26 ; 35)$ & $32(26 ; 37)$ & $28(24 ; 31)$ \\
MeanNN, ms & $810(732 ; 849)$ & $782(732 ; 835)$ & $776(708 ; 830)$ & $764(694 ; 832)$ \\
SDNN n (\%) & $16,9(13,6 ; 19,4)$ & $17,2(16,3 ; 21,1)$ & $13,1(11,3 ; 5,3)$ & $12,2(10,7 ; 14,6)$ \\
SDNNi n (\%) & $6,8(5,1 ; 8,1)$ & $6,8(5,0 ; 8,3)$ & $7,1(6,1 ; 7,7)$ & $5,2(4,9 ; 5,7)$ \\
RMSSD n (\%) & $2,1(1,5 ; 2,3)^{\star *}$ & $4,1(2,7 ; 5,2)^{\star *}$ & $1,7(1,4 ; 3,7)^{\star}$ & $2,8(2,2 ; 3,9)^{\star}$ \\
\end{tabular}

Data are presented in median values and interquartile range, ${ }^{*} \mathrm{p}<0,05,{ }^{* *} \mathrm{p}<0,005$ vs controls.

There was a significant negative correlation in BD patients between HRV (SDNNin $\%)$ and age $(r=-0,4 ; p=0,00)$, disease duration $(r=-0,3 ; p=0,00)$, BMI $(r=$ $-0,2 ; p<0,01)$, cholesterol levels $(r=-0,3 ; p=0,00)$, LDLP $(r=-0,3 ; p=0,00)$ and increased IMT ( $\mathrm{r}=-0,2 ; p=0,04)$, and also between HRV (RMSSD\%) and age $(r=-0,2 ; p=0,04)$, disease duration $(r=-0,2 ; p=0,01)$, cholesterol levels $(r=-0,3$; $p=0,00)$, HDLP $(r=-0,2 ; p=0,04)$; a positive correlation was established between HRV (SNNN\%) and smoking $(r=-0,2 ; p=0,04)$. The control group showed positive correlation between HRV (SNNN\%) and increased IMT ( $r=0,4 ; p=0,01)$. Conclusion: HRV reduction reflects impaired sympathetic -parasympathetic regulation in $\mathrm{BD}$ pts, associated with pts' age, disease duration and presence of traditional cardiovascular risk factors: BMI, increased cholesterol levels, LDLP, and such asymptomatic manifestation of atherosclerosis as increased IMT.

Disclosure of Interests: None declared

DOI: 10.1136/annrheumdis-2021-eular.3682

\section{POS0117 RENAL AND OVERALL OUTCOMES OF DOUBLE- POSITIVE (ANCA AND ANTI-GBM ANTIBODIES) PATIENTS COMPARED TO ANCA-ASSOCIATED VASCULITIS PATIENTS WITH SEVERE RENAL INVOLVEMENT: A MULTICENTER RETROSPECTIVE STUDY WITH SYSTEMATIC RENAL PATHOLOGY ANALYSIS}

M. Clerte ${ }^{1}$, R. Philip ${ }^{2}$, C. Levi ${ }^{1}$, E. Cornec-Legall ${ }^{3}$, V. Audard ${ }^{4}$, A. Huart ${ }^{5}$, X. Puéchal ${ }^{6}$, M. Touzot ${ }^{7}$, N. Rabot ${ }^{8}$, E. Thervet ${ }^{1}$, A. Aouba ${ }^{2}$, A. Karras ${ }^{1}{ }^{1}{ }^{1}$ Division of Nephrology, University Paris Descartes, Georges Pompidou European Hospital, APHP, 75, Paris, France; ${ }^{2}$ Division of Clinical Immunology and Internal Medicine, Normandie University, UNICAEN, CHU of Caen Normandie, 14, Caen France; ${ }^{3}$ Division of Nephrology, Hospital of the Cavale Blanche, CHRU of Brest, 29, Brest, France; ${ }^{4}$ Department of Nephrology and Renal Transplantation, Reference Center-Idiopathic Nephrotic Syndrome, Henri-Mondor Hospital, APHP F-94000 Créteil, INSERM U955, Paris East Créteil University F-94000, 94, Créteil, France; ${ }^{5}$ Division of Nephrology and Transplantation, Reference Center of Rare Renal Diseases, University Paul Sabatier - Toulouse III, Hôpital Rangueil, 31, Toulouse, France; ${ }^{6}$ Division of Clinical Immunology and Internal Medecine, University Paris Descartes, Cochin Hospital, APHP, 75014, 75, Paris, France; ${ }^{7}$ Division of Dialysis and Therapeutic Apheresis, Health Center of Aura Paris Plaisance, 75, Paris, France; ${ }^{8}$ Division of Immunology and Nephrology, University François Rabelais, CHRU of Tours, 37, Tours, France

Background: Among small vessel vasculitis, double-positive patients (DPP) combining serum and/or histologic findings for glomerular basement membrane (GBM) disease, and anti-neutrophil cytoplasmic antibodies (ANCA), is a rare newly and poorly described condition.

Objectives: We aimed to compare characteristics between DPP and ANCA-associated vasculitis patients (AAVP) with severe-renal-involvement.

Methods: Retrospective multicenter study comparing 33 DPP and 45 severe-renal-involvement (serum creatinine $>300 \mu \mathrm{mol} / \mathrm{L}$ ) AAVP, all with biopsy-proven nephropathy.

Results: Except for 2 patients (6\%) who had pure renal presentation during their entire follow-up period, others exhibited at least one extrarenal manifestation: pulmonary involvement (64\%), weight loss (39\%), gastrointestinal involvement (33\%), ENT manifestations (21\%), musculoskeletal symptoms $(21 \%)$, fever (18\%), neurological (12\%), cutaneous $(6 \%)$ and/or cardiac $(6 \%)$ signs. All DPP (including up to $18 \%$ exhibiting negative serum anti-GBM antibodies) presented severe acute kidney failure with histologic GBM involvement. Compared to our AAVP, they had higher serum creatinine $(719$ versus $501 \mu \mathrm{mol} / \mathrm{L} ; \mathrm{p}=0.006)$ and a higher of patients requiring initial renal replacement therapy ( $82 \%$ vs $36 \%$; $p<0.001)$. Berden classification significantly differed $(p=0.003)$, with more crescentic glomerulonephritis and fewer sclerotic lesions in DPP. One-year renal survival was significantly lower in DPP than in AAVP $(27 \%$ versus $64 \% ; p<0.0002)$. With comparable proportions of ANCA subtypes (2/3 with anti-MPO autoantibodies), 